\title{
Building Cleaning and Pest Control Workers
}

National Cancer Institute

\section{Source}

National Cancer Institute. Building Cleaning and Pest Control Workers. NCI Thesaurus. Code C122431.

Workers who keep buildings and their surrounding grounds in clean, orderly, and good condition, and/or control unwanted creatures. 